\title{
UMA VISÃO DA PRODUÇÃO CIENT ÍFICA INTERNACIONAL SOBRE A CLASSIFICAÇÃO INTERNACIONAL PARA A PRÁTICA DE ENFERMAGEM
}

\author{
Francine Dutra M ATT EIa, Rucieli M aria T ONIOLOb \\ Andreia M ALU CE LLI', M arcia Regina CU BA S ${ }^{d}$
}

\section{RESUMO}

A Classificação I nter nacional para a Prática de E nfermagem (CIPE ${ }^{\circledR}$ ) é um sistema classificatório que visa padronizar uma linguagem universal para Enfermagem. Este artigo propõe identificar os estudos desenvolvidos no âmbito mundial abordando $\mathrm{C} C \mathrm{PE}{ }^{\circledR}$, categorizando-os segundo suas final idades. T rata-se de uma revisão de literatura, em base de dados da Biblioteca Virtual em Saúde, pelo o termo "ICN P", com abrangência até 2009. Foram encontrados 124 artigos; 65 analisados, cujo conteúdo foi agrupado em nove categorias: abordagens gerais; aplicabilidade à prática; avaliação de classificações; experiências com recur sos computacionais; desenvolvimento e inclusão de termos; abordagem sobre sistemas classificatórios; uso para ancorar a construção de declaraç̧̃̃es de enfermagem; traduções; e outros. Verificou-se que poucos trabalhos apresentam projetos ou avaliam resultados de aplicações práticas da CIPE ${ }^{\circledR}$; a maioria aborda aspectos conceituais ou real iza comparações com outras classificações. D iversos trabal hos concluem sobre a adequação e rel evância da CIPE ${ }^{\circledR}$, mas apontam a necessidade de aperfeiçoamento.

D escritores: Processos de enfer magem. Classificação. Prática profissional.

\section{RESUMEN}

L a Clasificación Internacional para la P ráctica de E nfer mería (ICN P ${ }^{\circledR}$ ) tiene como objetivo estandarizar un lenguaje universal para la enfermería. T iene como objetivo discutir los resultados de estudios que abordan la ICN P $₫$. E s una revisión de la literatura en la base de datos de la Biblioteca Virtual en Salud, por el término "ICN P", para el año 2009. 124 artícul os encontrados; 65 se analizaron y seagruparon en nuevecategorías: enfoques generales; aplicabilidad a la práctica; la evaluación de las clasificaciones; la experiencia con los recursos de cómputo; el desar rollo y la inclusión de los términos; el enfoque delas clasificaciones; andlaje para el edificio de las declaraciones; traducciones; y otros. P ocos estudios han eval uado los resultados de los proyectos 0 aplicaciones de la ICN P $\circledast$, en la mayor parte se analizan cuestiones conceptuales 0 se hacen comparaciones con otras clasificaciones. Varios estudios concl uyen su pertinencia y relevancia, pero señalan la necesidad de mejora.

D escriptores: Procesos de enfermería. Clasificación. P ráctica profesional.

Título: P roducción científica sobre la Clasificación I nternacional para la P ráctica de E nfermería.

\section{ABST RACT}

Thel nter national Classification for $\mathrm{N}$ ursing P ractice (ICN P $\left.{ }^{\circledR}\right)$ is a classification system that aims at an universal standardized language for nursing. T his article aims to discuss the results of studies addressing ICN P ${ }^{\circledR}$. T his is a review of the literature in the database of the Virtual $\mathrm{H}$ ealth L ibrary, by the term "ICN P " with coverage until 2009. 124 articles were found; 65 were analyzed, and their contents were grouped in nine categories: general approaches; applicability in practice; assessment ratings; experiments with computational resources; development and inclusion of ter ms; approach to classification systems; use to anchor the building of nursing statements; translations; and others. It was found that few studies present projects or evaluate the practical applications of ICN ${ }^{\otimes}$. The majority discusses conceptual issues or compare ICN ${ }^{\otimes}$ with other classification systems. Several studies conclude about the appropriateness and relevance of ICN P ${ }^{\circledR}$, despitepointing a need for improvement.

Descriptors: $\mathrm{N}$ ursing process. Classification. P rofessional practice

Title: International scientific output on the International Classification for $\mathrm{N}$ ursing P ractice

\footnotetext{
a M estre em Tecnologia em Saúde, D ocente da Faculdade Pequeno Príncipe, Curitiba, Paraná, Brasil.

b M estranda pelo Programa de Pós-G raduação em Tecnologia em Saúde da Pontifícia U niversidade Católica do Paraná (PU CPR), Enfermeira do Hospital Vita Curitiba, Paraná, Brasil.

'D outora em Engenharia Eletrotécnica e de Computadores, D ocente do Programa de Pós-G raduação em Informática da PU CPR, Curitiba, Paraná, Brasil.

d D outora em Enfermagem, D ocente do Programa de Pós-G raduação em Tecnologia em Saúde da PU CPR, Curitiba, Paraná, Brasil.
} 


\section{INT RODUÇÃO}

0 conhecimento, conceitos e significados da E nfermagem e sua utilização na prática são objetos de questionamento. $\mathrm{Na}$ busca de sua identidade, ela desenvolveu conhecimentos capazes de garantir sua legitimidade, visibilidade e autonomia ${ }^{(1)}$. Entretanto, a ausência de uma linguagem universal que defina e descreva sua prática tem comprometido seu desenvolvimento como ciência(2).

Ciente deste cenário, o International Council of $\mathrm{N}$ urses (ICN) organiza a Classificação Internacional para a P rática de E nfermagem (CIPE $\left.{ }^{\circledR}\right)$, um sistema de classificação de termos cuja finalidade primária é padronizar uma linguagem universal para a Enfermagem, representando conceitos e cuidados de sua prática, permitindo comparação entre dados de difer entes populações e tempos ${ }^{(3)}$.

0 projeto da $\mathrm{CIPE}^{\circledR}$ foi concebido após a Organização M undial de Saúde (OM S) apontar a necessidade de que a prática de enfer magem fosse descrita mundialmente ${ }^{(4)}$. Considera-se a $\mathrm{CIPE}^{\circledR}$ um instrumento de informação capaz de fornecer dados que identifiquem a contribuição da profissão no cuidado da saúde e permitir mudanças práticas através de educação, administração e pesquisa ${ }^{(5)}$. Produz, também, informações para o processo decisório do enfermeiro, possibilitando a elaboração de diagnósticos, resultados e intervenções de enfermagem ${ }^{(6)}$.

A o longo dos anos, a CIPE ${ }^{\circledR}$ alterou sua estrutura, conteúdo e apresentação hierárquica dos termos. As versões Alfa (1996), a B eta (2000) ea B eta 2 (2001), eram experimentais e continham duas classificações: de fenômenos e de ações de enfermagem. A versão 1.0 (2005) apresentou modificações significativas, relacionadas à reestruturação do modelo multiaxial e inclusão de uma ontologia, recurso computacional que classifica conceitos hierarquicamente, viabilizando combinações e restrições entre ter mos. A versão 1.1 (2008) inova com a inclusão de diagnósticos e inter venções pré-combinadas e, em 2009, a versão 2.0 é apresentada em resposta ao ajuste necessário para inclusão da CIPE ${ }^{\circledR}$ na Família de Classificações Internacionais da OMS.

M uitos países se envolveram na elaboração da CIPE ${ }^{\circledR}$. No Brasil, a A ssociação Brasileira de Enfermagem (ABE n) desenvolveu, entre 1996 e 2000, 0 projeto da Classificação Internacional das Práticas deE nfer magem em Saúde Coletiva (CIPESC $\left.{ }^{\circledR}\right)$, que contribui com um inventário vocabular de termos utilizados na atenção básica(7).

0 ICN previa que a $\mathrm{CIPE}^{\circledR}$ fosse um instrumento de troca de informações, com potencial para identificar a contribuição da enfermagem nos cuidados de saúde e influenciar na elaboração de políticas de saúde. N este contexto, conhecer as publicações referentes ao tema pode auxiliar na discussão acerca do impacto da $\mathrm{CIPE}{ }^{\circledR}$, bem como possibilitar o desenvolvimento de pesquisas direcionadas às lacunas existentes.

0 objetivo deste trabalho é identificar os estudos desenvolvidos mundialmente abordando a CIPE ${ }^{\circledR}$, categorizando-os segundo suas finalidades.

\section{MÉT ODO}

T rata-se de uma revisão da literatura $a^{(8)}$ nas bases de dados $M$ edical $L$ iterature Analysis and Retrieval System Online (M EDLINE), Literatura L atino-A mericana e do Caribe em Ciências da Saúde (LILACS), Banco de Dados de Enfermagem (BDENF) e Scientific Electronic L ibrary Online (SciE LO), disponíveis na Biblioteca Virtual em Saúde (BVS), acessada pel o sítio <www.bireme.br>. U tilizou-se para a col eta o termo "I CN P" (International Classification of $\mathrm{N}$ ursing $\mathrm{P}$ ractice) e o universo de pesquisa abrangeu trabalhos publicados até 0 ano de 2009. A busca foi realizada entre novembro de 2009 a março de 2010.

Selecionaram-se trabalhos pelo título, resumo e sua pertinência ao objetivo da pesquisa, sem restrição ao tipo de estudo, forma de apresentação e idioma. Foram excluídos artigos em que a sigla "ICN P" tinha outro significado e os coincidentes em mais de uma base. Os resultados são apresentados na T abela 1, a seguir.

D os 124 trabalhos, 59 não estavam disponíveis em bancos institucionais (livres ou de acesso restrito) e destes, oito eram resumos em anais de congressos, cujo acesso se efetivava por compra. D esta forma, o universo contemplou 65 artigos.

Os artigos foram organizados em nove categorias temáticas: abordagens gerais sobre a $\mathrm{CIPE}{ }^{\circledR}$; aplicabilidade da $\mathrm{CI} P{ }^{\circledR}{ }^{\circledR}$ à prática de enfermagem; avaliação de sistemas classificatórios; experiências com recursos computacionais; desenvolvimento e inclusão de termos da $\mathrm{CIPE}{ }^{\circledR}$; abordagem geral sobre sistemas classificatórios; U so da CIPE ${ }^{\circledR}$ para ancorar a construção de declarações de enfermagem; traduções da CIPE ${ }^{\circledR}$; e outros trabal hos relacionados à CIPE ${ }^{\circledR}$. 
T abela 1 - Número de publicações encontradas pelo termo "ICNP" nas bases de dados MEDLINE, LILACS, BDENF e SCiE LO. N ov./ 2009 a mar./ 2010.

\begin{tabular}{lcccc}
\hline Base de dados & $\begin{array}{c}\text { Referências } \\
\text { encontradas }\end{array}$ & $\begin{array}{c}\text { Trabalhos } \\
\text { selecionados }\end{array}$ & $\begin{array}{c}\text { Trabalhos } \\
\text { coincidentes }\end{array}$ & $\begin{array}{c}\text { Trabalhos } \\
\text { considerados }\end{array}$ \\
\hline M ED L INE & 123 & 119 & - & 119 \\
LILACS & 14 & 12 & 8 & 4 \\
BDENF & 10 & 9 & 9 & - \\
SciE L O & 9 & 9 & 8 & 1 \\
\hline Total & $\mathbf{1 5 6}$ & $\mathbf{1 4 9}$ & $\mathbf{2 5}$ & $\mathbf{1 2 4}$ \\
\hline
\end{tabular}

\section{RESULTADOS E DISCUSSÃO}

\section{Identificação das produções}

Considerando as 124 referências, percebe-se que a partir de 1996, ano da primeira publicação da $\mathrm{CIPE}{ }^{\circledR}$, houve tendência de crescimento da quantidade de trabal hos, com pico em 2006 (F i-gura 1). Os dois trabal hos anteriores à ver são Alfa relatam o projeto e a intenção da classificação.

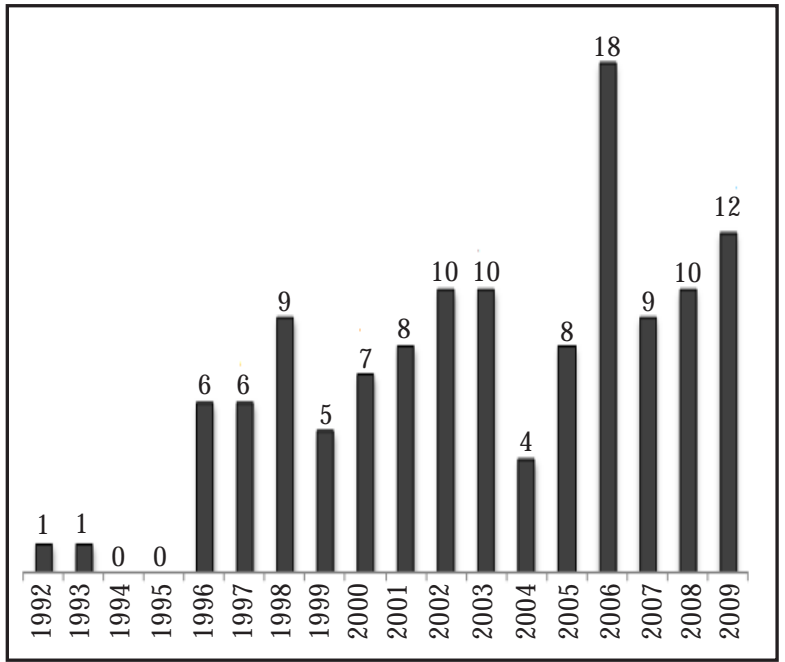

Figura 1 - T rabalhos sobre a CIPE ${ }^{\circledR}$ nas bases de dados M ED L INE LILACS, BDENF e SciELO, por ano de publicação. Nov./2009 a mar./ 2010.

Q uanto ao país de origem, os trabal hos foram classificados pelo local de produção, à exemplo dos de autoria do ICN, que foram classificados como Suíços. Verifica-se predominância de países do continente $E$ uropeu e destaca-se a quantidade de artigos brasileiros, semelhante aos norte-americanos (F igura 2). Recente pesquisa localizou 50 estudos brasileiros sobre a CIPE ${ }^{\circledR}$ (26 não indexados, obtidos manualmente), com hegemonia de estudos descritivos correlacionais quantitativos, voltados ao modelo clínico-individual e pesquisas relatando seu uso, além da contribuição da ABE n com o projeto CIPESC ${ }^{\circledR(9)}$.

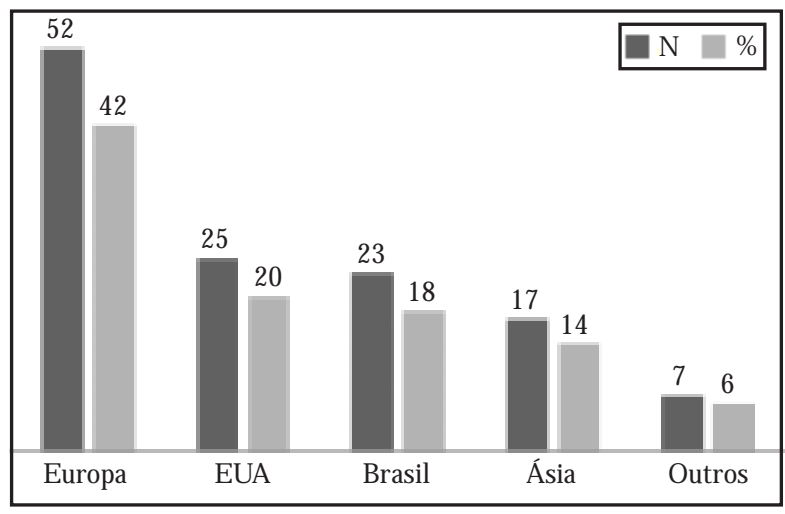

Figura 2 - Produção sobre a $\mathrm{CIPE}{ }^{\circledR}$ nas bases de dados M ED L INE, LILACS, BDE N F eSciE L O distribuída por país ou continente. N ov./ 2009 a mar./ 2010.

\section{Abordagens gerais sobre a CIPE ${ }^{\circledR}$}

Dos 65 artigos analisados, 22 apresentam a CIPE ${ }^{\circledR}$, descrevendo ou explicando conceitos ou temas correlacionados. Os artigos publicados entre 1992 e 1998, têm autoria predominante de consultores do ICN , participantes dos estudos que or iginaram a CIPE ${ }^{\circledR}$, dentre elas, as enfer meiras A my Coenen, J une Clark, N orma L ang, Randi M ortensen, $\mathrm{G}$ unnar $\mathrm{N}$ iel sen e $\mathrm{M}$ adeline $\mathrm{W}$ ake.

São textos que explicam a arquitetura multiaxial da $\mathrm{CIPE}{ }^{\circledR}$, demonstrando as diferenças com uma estrutura monoaxial e justificam a não necessidade de construir uma classificação independente para expressar resultados de enfermagem $^{(10,11)}$; que descrevem o Telenurse, projeto cujo objetivo foi promover 0 uso da $\mathrm{CIPE}^{\circledR}$ na Euro$\mathrm{pa}^{(12)}$; e abordam os trabal hos iniciais de construção da $\mathrm{CIPE}{ }^{\circledR}$, esclarecendo dúvidas, como as questões sobre a participação dos enfermeiros em seu desenvolvimento ${ }^{(13-25)}$. 
A partir de 2000, surgem trabal hos com abordagens mais distintas, porém restritos aos de aspectos gerais da $\mathrm{CIPE}^{\circledR}$, dentre eles: 0 que apresenta características dos Centros $\mathrm{CIPE}^{\circledR}$ e descreve o centro brasileiro, no estado da Paraíba ${ }^{(26)}$; relato a respeito de sistemas classificatórios de enfermagem, com três experiências da implantação da CIPE ${ }^{\circledR}$ no Brasil(2); e o que exemplifica a utilidade da CIPE ${ }^{\circledR}$ nos campos da prática clínica, da pesquisa e da administração e formulação de políticas de saúde ${ }^{(27)}$.

Outros artigos elaborados nesta ótica contribuíram para modificações na forma e no conteúdo da $\mathrm{CIPE}{ }^{\circledR}$ no que se refere ao eixo "Julgamento"(28); para estrutura de trabalho de validação dos termos a serem submetidos ao ICN ${ }^{(29)}$ e para sugestões de reorganização da versão $B$ eta ${ }^{(30)}$.

\section{A plicabilidade da CIPE ${ }^{\circledR}$ à prática de enfermagem}

Seis trabalhos apresentam mapeamentos cruzados entre termos da CIPE ${ }^{\circledR}$ e dados registrados, de forma não padronizada, em prontuários. U ma pesquisa conduzida no Brasil coletou dados de consultas de enfermagem de um serviço de planejamento familiar, cujos fenômenos descritos nos prontuários foram comparados com os termos da CIPE ${ }^{\circledR}$, mostrando alto grau de concordância ${ }^{(31)}$.

Dois estudos italianos, realizados durante 0 processo de tradução da versão em inglês para 0 idioma italiano, concluem que a $\mathrm{CIPE}^{\circledR}$ tem que evoluir, mas contribui para maior visibilidade da enfer magem. 0 primeiro analisou mais de três mil registros de 90 pacientes, e o segundo utilizou 1.071 registros relativos a 30 pacientes, tendo sido identificados 41 diagnósticos de enfermagem utilizando termos da CIPE ${ }^{\circledR}$ no primeiro estudo e 92 diagnósticos no segundo(32).

E studo sueco avaliou prontuários de 56 pacientes de uma unidade de doenças infecciosas, contendo dados sobre nutrição e cuidados com a pele. Verificou que a maior parte dos fenômenos e intervenções de enfermagem pôde ser expressa utilizando a CIPE ${ }^{\circledR}$ e concluiu que a classificação carece de desenvolvimento para abranger a atuação da enfer magem em sua totalidade $\mathrm{e}^{(33)}$.

A inda relacionado à avaliação de prontuários, foi verificada compatibilidade da CIPE ${ }^{\circledR}$ ver são Alfa com fenômenos/ intervenções encontrados em prontuários de 59 pacientes internados em um hospital de São Paulo(34); na N oruega, avaliou-se a compatibilidade da versão $B$ eta com fenômenos/ inter venções encontrados em prontuários de 30 pacientes, relacionados aos temas "circulação" e "eliminação" (35); em T aiwan, dados de registros de pacientes foram col etados e mapeados com os termos da CIPE ${ }^{\circledR}$, mostrando al ta concordância ${ }^{(36)}$.

\section{Avaliação de sistemas classificatórios}

A aplicabilidade de sistemas classificatórios de enfermagem, bem como a compatibilidade entre diferentes classificações, utilizando a CIPE ${ }^{\circledR}$ como foco principal ou como referência para avaliar outros sistemas, também foi objeto de estudo. N essa linha encontraram-se três grupos de conclusões: a compatibilidade e potencialidade da $\mathrm{CIPE}^{\circledR}$ em relação a outros sistemas classificatórios; a maior adequação de outra taxonomia; e a sugestão de melhorias.

Dentre o primeiro grupo, seis trabalhos foram consider ados relevantes: o mapeamento cruzado entre os termos da $\mathrm{CIPE}^{\circledR}$ (versão B eta 2 ) e do $E$ uropean $N$ ursing CareP athways (EN P) que concluiu que $98 \%$ das declarações do E N P podem ser reescritas utilizando a $\mathrm{CIPE}^{\circledR(37) ; 0}$ mapeamento cruzado entre a taxonomia da $\mathrm{N}$ orth A merican $\mathrm{N}$ ursing Diagnosis Association (NANDA ${ }^{\circledR}$ ), juntamente com a classificação de intervenções $N$ ursing Interventions Classification ( $\mathrm{NIC}$ ), a $\mathrm{H}$ ome $\mathrm{H}$ ealth Care Classification ( $\mathrm{HHCC}$ ) e a Community $\mathrm{H}$ ealth System (OM A HA), com os termos da CIPE ${ }^{\circledR}$, o qual concluiu que a $\mathrm{CIPE}^{\circledR}$ tem potencial para ser um sistema unificado de linguagem de Enfermagem e pode ser usado para descrever a maior parte das terminologias existentes ${ }^{(38)}$; 0 que realizou avaliação dos eixos de inter ven ção da $\mathrm{CIPE}{ }^{\circledR}$, comparando-os com os sistemas H HCC, Systematized N 0menclature of $M$ edicine-Clinical T erms - Reference Terminology (SN OM ED-RT) e Patient Care D ata Set (PCDS) ${ }^{(39)}$; o que avaliou o sistema Canadian $\mathrm{H}$ ealth O utcomes for B etter Information and Care (C$\mathrm{HOBIC}$ ) mapeando seus termos com a CIPE ${ }^{\circledR} 1.0$, concluindo que aquele sistema propicia informações clínicas padronizadas, consistentes e interoperáveis ${ }^{(40)} ; 0$ que procurou traduzir e integrar conceitos do sistema Clinical Care Classification (CCC) aos termos da $\mathrm{CIPE}^{\circledR} 1.0$, concluiu que ambos têm alto grau de compatibilidade ${ }^{(41)}$; e o que coletou dados de avaliações de enfermagem de quatro estabelecimentos de saúde nos Estados U ni- 
dos, mapeando os termos encontrados com a $\mathrm{CIPE}{ }^{\circledR}$, e posteriormente procedendo à sua validação com a taxonomia de diagnósticos da NANDA ${ }^{\circledR}$, concluindo que a estrutura da $\mathrm{CIPE}^{\circledR}$ a torna flexível e robusta, capaz de servir como modelo de representação para registros informatizados de avaliações de enfermagem ${ }^{(42)}$.

N este ponto surge divergência, pois outro artigo que avaliou diversos sistemas classificatórios everificou seu enquadramento em determinados critérios, conclui que a NANDA ${ }^{\circledR}$ é mais adequada(43).

A sugestão de melhorias foi objeto de trabalho que mapeou diagnósticos entre a taxonomia da NANDA ${ }^{\circledR}$, os termos da CIPE ${ }^{\circledR}$ e o sistema SN OM ED -CT, considerando que todas as três terminologias careciam de adequações ${ }^{(44)}$.

\section{Experiências com recursos computacionais}

Dois artigos apresentam trabalhos de utilização da linguagem GALEN Representation and Integration L anguage (GRAIL) para representar intervenções ${ }^{(45)}$ e diagnósticos ${ }^{(46)}$ de enfermagem baseados na $\mathrm{CIPE}^{\circledR}$. U m estudo avaliou um sistema baseado na $\mathrm{CIPE}{ }^{\circledR}$, denominado $\mathrm{N}$ ursing $\mathrm{D}$ ata Dictionary (NDD), voltado à elaboração de prontuários eletrônicos ${ }^{(47)}$. Outro trabalho procurou desenvolver uma ontologia para facilitar a descrição da prática de enfermagem, partindo do sistema denominado $\mathrm{N}$ ursing $\mathrm{M}$ aster, mapeando seus ter mos com a CIPE ${ }^{\circledR(48)}$. N esta categoria de trabal hos, estudo sul-coreano apresenta um mapeamento cruzado de termos e um sistema informatizado para construção de diagnósticos baseados na CI PE ${ }^{\circledR(49)}$.

\section{D esenvolvimento e inclusão de termos da CIPE $^{\circledR}$}

Outros trabalhos buscam atingir o objetivo de avaliar ou desenvolver termos da $\mathrm{CIPE}^{\circledR}$ por meio de pesquisas envolvendo profissionais de enfermagem. Investigação com enfermeiros da Índia a partir de questionário da $\mathrm{CIPE}{ }^{\circledR}$ sobre o termo "morte digna" colaborou com a revisão da definição deste termo na versão 1.1 $1^{(50)}$; outro estudo aplicou o questionário sobre "morte digna" da CIPE ${ }^{\circledR}$ a enfermeiros de diversos países, ressaltando as diferenças culturais encontradas(51); e um trabalho descreve a aplicação a 356 enfermeiras do Japão de questionário a respeito do termo "febre" queintegrou a validação da CIPE ${ }^{\circledR}$ versão Alfa ${ }^{(52)}$.
Quanto à inclusão de termos à $\mathrm{CIPE}{ }^{\circledR}$, no estado brasileiro da Paraíba, foi realizada uma pesquisa com 33 crianças de creches municipais, com o objetivo de avaliar a presença do fenômeno "violência". Os autores defendem que este fenômeno deve estar presente na classificação ${ }^{(53)}$. Este mesmo termo foi objeto de um artigo que descreve a metodologia e os resultados do encontro, ocorrido em 1997, entre dez especialistas de nove países com o objetivo de avaliar o fenômeno "violência" e elaborar sugestões para a melhoria de próximas versões da CIPE ${ }^{\circledR(54)}$. E m estudo sul-africano se descreve o processo de validação do termo "acampamento informal" (em inglês: informal settle ment) e sua submissão para inclusão na CIPE ${ }^{\circledR(55)}$.

\section{Abordagem geral sobre sistemas classificatórios}

A lguns artigos encontrados não têm a $\mathrm{CIPE}{ }^{\circledR}$ como tema principal, mas tratam de assuntos correlatos, com inserção da mesma. U m aborda diversas questões relacionadas aos "diagnósticos de enfermagem", reforçando a importância de uma padronização para a melhora da qualidade e da visibilidade da enfermagem ${ }^{(56)}$. O utro artigo aborda 0 desenvolvimento de terminologias de enfermagem, analisando alguns aspectos da evolução das taxonomias NAN DA ${ }^{\circledR}$ e CIPE ${ }^{\circledR}$ e posteriormente, avalia estes sistemas com base em critérios estabelecidos pelo Computer-based Patient Record Institute $(C P R I)^{(57)}$. U m trabalho aborda o desenvolvimento de um sistema classificatório específico para diagnósticos na área de cuidados intensivos, denominado Intensive Care $\mathrm{N}$ ursing $\mathrm{S}$ coring System (ICNSS) ${ }^{(58)}$. Outro texto descreve diversas iniciativas mundiais relacionadas ao desenvolvimento de terminologias de enfermagem ${ }^{(59)}$.

\section{U so da CIPE ${ }^{\circledR}$ para ancorar a construção de declarações de enfermagem}

Nesta categoria, pesquisadores da Polônia separaram dois grupos de enfermeiros, um dos quais formulou diagnósticos livremente e o outro utilizou ter mos da $\mathrm{CIPE}{ }^{\circledR}$; os resultados demonstram que a $\mathrm{CIPE}^{\circledR}$ é compatível com os diagnósticos livres ${ }^{(60)}$; outro estudo adotou a $\mathrm{CIPE}^{\circledR}$ para planejar 0 atendimento de paciente com Lúpus e concluiu que a $\mathrm{CIPE}^{\circledR}$ facilita o planejamento da assistência(61); e um artigo descreve o processo de implantação da $\mathrm{CIPE}^{\circledR}$ em hospitais do Paquistão(62). 
Outros três artigos relatam pesquisas destinadas à construção de declarações de enfermagem, todos brasileiros. Um dos trabalhos apresenta a construção de intervenções de enfermagem baseadas na $\mathrm{CIPE}{ }^{\circledR}$ para os diagnósticos de enfermagem mais freqüentes em dois serviços públicos de assistência a saúde da mulher ${ }^{(1)}$; outro estudo desenvolveu uma nomenclatura para intervenções de enfermagem com base nos termos da CIPE ${ }^{\circledR(63)}$; e outro artigo descreve a reestruturação da aplicação do processo de enfer magem em uma U nidade de Terapia Intensiva, utilizando a CIPE ${ }^{\circledR}$ para a construção de diagnósticos de enfermagem ${ }^{(64)}$.

$\mathrm{N}$ esta categoria se incluiu estudo realizado com graduandos e docentes de enfermagem que concluiu que a $\mathrm{CIPE}^{\circledR}$ facilita a implementação do processo de enfermagem e contribui para 0 desenvolvimento do raciocínio clínico(65).

\section{T raduções da $\mathrm{CIPE}^{\circledR}$}

A tradução foi assunto de dois artigos. U m aborda as metodologias utilizadas para tradução de sistemas classificatórios, concluindo pela maior adequação da back-translation, e descreve a tradução da CIPE ${ }^{\circledR}$ para o português ${ }^{(66)}$ e outro descreve 0 processo de tradução dos fenômenos de enfermagem do ICN P versão Alfa para o português ${ }^{(67)}$.

\section{O utros trabal hos relacionados à $\mathrm{CIPE}^{\circledR}$}

N este grupo, restaram cinco artigos cujos temas diversos não justificam a formação de uma categoria: uma avaliação da $\mathrm{CIPE}{ }^{\circledR}$ com base em critérios estabelecidos pelo Computer-based Patient R ecord Institute (CPRI), comparando-a com outros sistemas classificatórios reconhecidos pela A merican N urses Association (ANA) (68); uma avaliação da conformidade da aplicação de uma norma International Organization for Standardization (ISO) específica para elaboração de terminologias de enfermagem, na construção da CIPE ${ }^{\circledR} 1.0^{(69)}$; uma revisão de literatura dos conceitos "autonomia" e"necessidades de saúde" e suas aplicações na implantação do processo de enfer magem, concluindo que estes termos são mais bem descritos na $\mathrm{CIPE}^{\circledR} \mathrm{e}$ CIPESC ${ }^{\circledR(70) ;}$; um estudo que identificou diferenças e semel hanças entre termos da $\mathrm{CIPE}^{\circledR} 1.0$, com a versão B eta 2 e com a CIPE SC ${ }^{\circledR(5)}$; e, finalmente, uma revisão bibliográfica sobre sistemas classificatórios, que conclui que a maioria dos estudos baseia- se no modelo clínico-individual, poucos com enfoque no coletivo, e destes a maior parte é divulgada pela $A B E n^{(71)}$.

\section{CONSIDERAÇÕES FINAIS}

T orna-se evidente que os objetivos estratégicos da CIPE ${ }^{\circledR}$ vêm sendo alcançados, pois se percebe a preocupação em mantê-la atual izada e sustentar este processo. F ica clara sua utilização pelas comunidades internacionais e sua compatibilidade com outras classificações e padronizações. A spectos estes, que permitem a realização de pesquisas que garantam seu desenvolvimento contínuo, assegurando a contribuição para crescimento e visibilidade da profissão.

N este contexto, é importante ressaltar a participação brasileira na produção de conhecimento sobre 0 assunto.

Constata-se a incipiência de pesquisas com objetivo de avaliar a aplicabilidade da CIPE ${ }^{\circledR}$. A maioria dos artigos se limita a abordar aspectos conceituais, apresentar o sistema ou comparar a CIPE ${ }^{\circledR}$ com outros sistemas classificatórios.

Os estudos que avaliam a $\mathrm{CIPE}^{\circledR}$ concluem que o sistema é viável e que pode contribuir, com ressalvas de que são necessários aperfeiçoamentos. Deve-se considerar que o desenvolvimento permanente é uma característica intrínseca de um sistema como a $\mathrm{CIPE}{ }^{\circledR}$, e que quanto mais disseminada estiver sua utilização, mais rapidamente adquirirá consistência.

Há um longo percurso para que a $\mathrm{CIPE}^{\circledR}$ seja reconhecida pelos enfermeiros como um sistema classificatório útil para a prática e valorização da profissão, porém todos os estudos encontrados ratificam que o caminho está correto.

\section{REFERÊ NCIAS}

1 G erk M AS, Barros SM O. Intervenções de enfermagem para os diagnósticos de enfermagem mais frequentes em dois ser viços públicos de assistência à saúde da mulher. Acta Paul Enferm. 2005;18(3):260-8.

2 Nóbrega M M L, Garcia TR. Perspectivas de incorporação da Classificação I nternacional para a P rática deE nfermagem (CIPE ${ }^{\circledR}$ ) no Brasil. Rev Bras Enferm. 2005;58(2):227-30.

3 Silva KL, Cruz DSM , Furtado LG, M angueira So, Albuquerque CC, N obrega M M L . Classificação I nter- 
nacional para a Prática de E nfermagem - CIPE ${ }^{\circledR}$. In: Nóbrega M M L, Silva KL, organizadoras. F undamentos do cuidar em enfer magem. J oão Pessoa: I mprima; 2007. p. 221-41.

4 Comitê Inter nacional de Enfermeiros. CIPE ${ }^{\circledR}$ Versão 1: Classificação Internacional para a Prática de Enfermagem. São Paulo: Algol; 2007.

5 Garcia TR, Egry EY, organizadores. Integralidade da atenção no SU S e sistematização da assistência de enfermagem. Porto A legre: A rtmed, 2010.

6 Silva RR, M alucelli A, Cubas M R. Classificações de enfermagem: mapeamento entre termos do foco da prática. Rev Bras Enferm. 2008;61(6):835-40.

7 Egry EY, Antunes M J M, Lopes M GD. Projeto ClPESC CIE-ABEn. In: G arcia T R, Egry EY, organizadores. I ntegralidade da atenção no SU S e sistematização da assistência de enfermagem. Porto Aleg re: Artmed; 2010. p. 175-91.

8 Rother $\mathrm{ET}$. Revisão sistemática $X$ revisão narrativa [ editorial] . Acta Paul Enferm. 2007;20(2):v-vi.

9 M azoni SR, Rodrigues CC, Santos D S, Rossi LA, Carvalho EC. Classificação Internacional para a Prática de Enfer magem e a contribuição brasileira. Rev Bras Enferm. 2010;63(2):285-9.

10 Nielsen GH, M ortensen RA. The architecture of ICN P: time for outcomes: part I. I nt N urs Rev. 1997; 44(6):182-8.

$11 \mathrm{~N}$ ielsen GH, M ortensen RA. The architecture of ICN P: time for outcomes: part II. Int N urs Rev. 1998; 45(1):27-31.

12 ICN P in Europe: T elenurse. Int N urs Rev. 1996; 43 (6):188-9.

13 Clark J, Lang N. N ursing's next advance: an internal classification for nursing practice. Int N urs Rev. 1992;39(4):109-11.

14 W ake M M , M urphy M, Affara FA, L ang N M, Clark J, M ortensen R. T oward an International Classification for Nursing Practice: a literature review \& survey. Int N urs Rev. 1993;40(3):77-80.

15 Coenen A, Wake M. Developing a database for an International Classification for Nursing Practice (ICN P). Int N urs Rev. 1996;43(6):183-7.
16 Nielsen GH, M ortensen RA. The architecture for an International Classification for $\mathrm{N}$ ursing P ractice (ICN P). Int N urs Rev. 1996;43(6):175-82.

17 Clark DJ. How nurses can participate in the development of an ICNP. Int N urs Rev. 1996;43(6):171-4.

18 Introducing ICN's International Classification for N ursing Practice (ICN P): a unifying framework. Int N urs Rev. 1996;43(6):169-70.

19 Clark J, L ang N . T he International Classification for N ursing Practice (ICNP): nursing outcomes. Int N urs Rev. 1997;44(4):121-4.

20 W ake $M$, Coenen A. N ursing diagnosis in the International Classification for N ursing Practice (ICN P). N urs Diagn. 1998;9(3):111-8.

21 Warren JJ, Coenen A. International classification for nursing practice (ICN P): most-frequently asked questions. J Am M ed Inform Assoc. 1998;5(4):335-6.

22 Joel LA. From NANDA to ICNP. A m J N urs. 1998; 98(7):7.

23 Coenen A. Building a unified nursing language system: the ICN P. Int N urs Rev. 2003;50(2):65-6.

24 Conrick $M$. The international classification for nursing practice: a tool to support nursing practice? Collegian. 2005;12(3):9-13.

25 Simpson RL . ICN P: the language of worldwidenursing. N urs M anage. 2007;38(2):15, 18.

26 G arcia T R, N óbrega M M , Coler M S. Centro CIPE ${ }^{\circledR}$ do Prog rama de Pós-G raduação em E nfermagem da UF PB. Rev Bras Enferm. 2008;61(6):888-91.

27 Baernholdt M, Lang N M. W hy an ICN P ? Links among quality, information and policy. Int N urs Rev. 2003;50(2):73-8.

28 Coler M S. Reflections on the judgement Axis, ICN P beta. Int N urs Rev. 2003;50(1):15-21.

29 Feringa M M , G oossen W T, Coenen A. Submitting terms to the inter national classification for nursing practice (ICN P). Int N urs Rev. 2002;49(3):154-60.

30 Kisilowska M. Reorganized structure and other proposals for the ICN P development. International Classification for Nursing Practice. Int Nurs Rev. 2001;48(4):218-23. 
31 Camiá G E, Barbieri M , M arin H de F. Fenômenos de enfermagem identificados em consultas de planejamento familiar segundo a ICN P - ver são Beta 2. Rev Lat Am Enfermagem. 2006;14(5):674-81.

32 Sansoni J, G iustini M . M ore than terminology: using ICN P to enhance nursing's visibility in I taly. Int N urs Rev. 2006;53(1):21-7.

33 Ehnfors M , F lorin J, Ehrenberg A. A pplicability of the Inter national Classification of N ursing Practice (ICNP) in the areas of nutrition and skin care. Int J N urs Terminol Classif. 2003;14(1):5-18.

34 Cruz D M , Gutierrez BA, López AL, Souza T T, Assami $S$. Congruence of terms between lists of problems and the ICN P: Alpha version: International Classification for Nursing Practice. Int Nurs Rev. 2000;47(2):89-96.

35 Ruland CM . E valuating the Beta version of the International Classification for Nursing Practice for domain completeness, applicability of its axial structure and utility in clinical practice: a N orwegian project. Int N urs Rev. 2001;48(1):9-16.

$36 \mathrm{Kuo} \mathrm{CH}$, Yen M. Cross-mapping ICN P terms with Taiwanese gynecological nursing records. J Nurs Res. 2006;14(4):271-8.

37 W ieteck P. Furthering the development of standardized nursing terminology through an ENP-ICN P cross-mapping. Int N urs Rev. 2008;55(3):296-304.

$38 \mathrm{H}$ yun S, Park H A. Cross-mapping the ICN P with NAN D A, H HCC, O maha System and N IC for unified nursing language system development. Int N urs Rev. 2002;49(2):99-110.

39 Bakken S, Parker J, Konicek D, Campbell KE. An evaluation of ICNP intervention axes as terminology model components. Proc A M IA Symp. 2000:42-6.

$40 \mathrm{H}$ annah KJ, W hite PA, Nagle L M , Pringle D M. Standardizing nursing information in Canada for inclusion in electronic health records: C-HOBIC. J Am M ed Inform A ssoc. 2009;16(4):524-30.

41 M atney SA, DaD amio R, Couderc C, D lugos M, Evans J, Gianonne $G$, et al. T ranslation and integration of CCC nursing diagnoses into ICN P.J A m M ed Inform Assoc. 2008;15(6):791-3.

42 Dykes PC, Kim HE, Goldsmith D M, Choi J, Esumi $\mathrm{K}, \mathrm{G}$ oldberg HS. T he adequacy of ICN P version 1.0 as a representational model for electronic nursing assessment documentation. J A m M ed Inform Assoc. 2009;16(2):238-46.

43 M üller-Staub M , L avin M A, N eedham I, van A chterberg T. M eeting the criteria of a nursing diagnosis classification: evaluation of ICN P, ICF, NANDA and ZE F P. Int J N urs Stud. 2007;44(5):702-13.

$44 \mathrm{H}$ ardiker N R, Casey A, Coenen A, Konicek D. M utual enhancement of diverseterminologies. A M IA Annu Symp Proc. 2006:319-23.

$45 \mathrm{H}$ ardiker $\mathrm{NR}$, Rector AL. M odeling nursing terminology using the GRAIL representation language. J Am M ed Inform A ssoc. 1998;5(1):120-8.

$46 \mathrm{H}$ ardiker NR, Rector AL. Structural validation of nursing terminologies. J A m M ed Inform Assoc. 2001;8(3):212-21.

$47 \mathrm{Cho} \mathrm{I}$, Park HA. Evaluation of the expressiveness of an ICNP-based nursing data dictionary in a computerized nursing record system. J Am M ed Inform Assoc. 2006;13(4):456-64.

48 J iang $G$, Sato H, Endoh A, Ogasawara K, Sakurai T. An ontological approach to support the description of nursing practice in Japan with the ICNP. Int J M ed Inform. 2007;76(1):55-65.

49 Cho I, Park H A. D evelopment and eval uation of a terminology-based el ectronic nursing record system. J Biomed Inform. 2003;36(4/ 5):304-12.

50 D oorenbos AZ, W ilson SA, Coenen A, Borse N N. Dignified dying: phenomenon and actions among nurses in India. Int N urs Rev. 2006;53(1):28-33.

51 D oorenbos AZ, W ilson SA, Coenen A. A cross-cultural analysis of dignified dying. J Nurs Scholarsh. 2006;38(4):352-7.

52 I kematsu Y. Characteristics of and interventions for fever in Japan. Int N urs Rev. 2004;51(4):229-39.

53 Coler M S, A raújo LC, Coêlho AA, Figueiredo T M, Freire M R, M oreira M E. Social violence: a case for classification as a sub-phenomenon of community in the ICN P. Int N urs Rev. 2000;47(1):8-18.

54 Coler M S. Building an intercultural nursing terminology bank for the phenomenon, Violence, of the International Classification of Nursing Practice: a methodological perspective. Int Nurs Rev. 2001;48 (2):93-101. 
55 G eyer N, M muwe-H lahane S, Shongwe-M agongo $R G$, Uys E. Contributing to the ICN P: validating the term 'informal settlement'. Int Nurs Rev. 2005; 52(4):286-93.

56 Hogston R. Nursing diagnosis and classification systems: a position paper. J Adv N urs. 1997; 26(3): 496-500.

57 M oen A, H enry SB, W arren JJ. Representing nursing judgements in the electronic health record. J Adv Nurs. 1999;30(4):990-7.

58 Pyykkö AK, L aurila J, Ala-Kokko TI, Hentinen M, Janhonen SA. Intensive care nur sing scoring system: part 1: classification of nursing diagnoses. Intensive Crit Care N urs. 2000;16(6): 345-56.

59 Coenen A, Marin HF, Park HA, Bakken S. Collaborative efforts for representing nursing concepts in computer-based systems: inter national per spectives. J Am M ed Inform Assoc. 2001;8 (3):202-11.

60 Zarzycka D, Górajek-Józwik J. N ursing diagnosis with the ICNP in the teaching context. Int Nurs Rev. 2004;51(4):240-9.

61 Bittencourt GK, Beserra PJ, Nóbrega M M . Assistência de enfermagem a paciente com lupus eritematoso sistêmico utilizando a CIPE ${ }^{\circledR}$. Rev Gaúcha Enferm. 2008;29(1):26-32.

62 Rukanuddin RJ. Introduction and development of NCP using ICN P in Pakistan. I nt N urs Rev. 2005;52 (4):294-303.

63 L ima CL , N óbrega M M . N omenclatura de intervenções de enfermagem para clínica médica de um hospital escola. Rev Bras Enferm. 2009; 62(4):570-8.
64 T ruppel T C, M eier M J, Calixto RC, Peruzzo SA, Crozeta K. Sistematização da assistência de enfermagem em U nidade de T erapia I ntensiva. Rev Bras E nferm. 2009;62(2):221-7.

65 P feilsticker D C, Cadê N V. Classificação I nternacional para a Prática de E nfermagem: significados atribuídos por docentes e graduandos de enfer magem. Rev Enferm UERJ. 2008;16(2): 236-242.

66 N óbrega M M L, G utiérrez M G R. M étodo utilizado na adaptaçäo transcultural da Classificaçäo de F enômenos de Enfermagem da CIPE : versão Alfa. Acta Paul Enferm. 2001;14(3):44-51.

67 Nóbrega M M L, Gutierrez M G. Semantic equivalence of the $\mathrm{N}$ ursing $\mathrm{P}$ henomena Classification of ICNP: Alpha version in Brazilian Portuguese. Int N urs Rev. 2000;47(1):19-27.

68 H enry SB, Elfrink V, M cN eil B, W arren J. T helCN P's relevance in the US. Int N urs Rev. 1998; 45(5):1539.

$69 \mathrm{H}$ ardiker NR, Coenen A. Interpretation of an international terminology standard in the development of a logic-based compositional terminology. Int J M ed Inform. 2007;76 Suppl 2:S274-80.

70 Barros D G, Chiesa AM . A utonomia e necessidades de saúde na Sistematização da A ssistência de E nfermagem no olhar da saúde coletiva. Rev Esc Enferm U SP. 2007;41(n esp):793-8.

71 Cubas M R, E gry EY. Classificação I nternacional de Práticas de Enfermagem em Saúde Coletiva CIPESC. Rev Esc Enferm USP. 2008;42 (1):1816.

Recebido em: 29/ 03/ 2011

A provado em: 29/ 11/ 2011

\author{
Endereço da autora / Dirección del autor / \\ Author's address: \\ M arcia Regina Cubas \\ Pontifícia U niversidade Católica do Paraná \\ Centro de Ciências Biológicas e da Saúde \\ Programa de Pós-G raduação em T ecnologia \\ em Saúde \\ Rua I maculada Conceição, 1155 \\ 80215-901, Curitiba, PR \\ E-mail:m.cubas@pucpr.br
}

